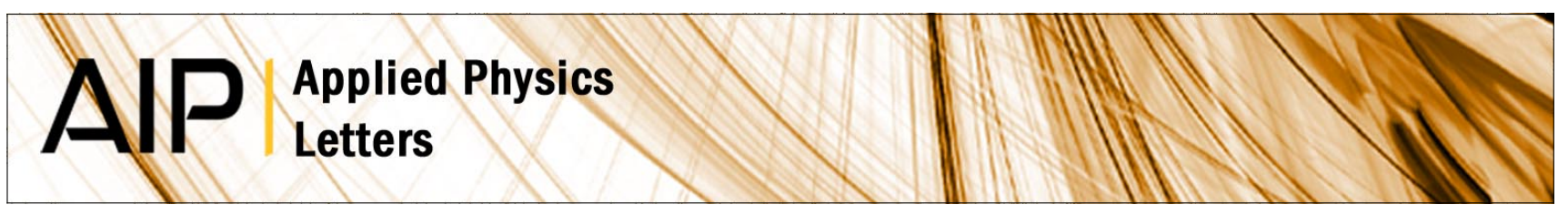

\title{
Spin-flip phenomena at the Co|graphene|Co interfaces
}

Baihai Li, Liang Chen, and Xiaoqing Pan

Citation: Appl. Phys. Lett. 98, 133111 (2011); doi: 10.1063/1.3571553

View online: http://dx.doi.org/10.1063/1.3571553

View Table of Contents: http://apl.aip.org/resource/1/APPLAB/v98/i13

Published by the AIP Publishing LLC.

Additional information on Appl. Phys. Lett.

Journal Homepage: http://apl.aip.org/

Journal Information: http://apl.aip.org/about/about_the_journal

Top downloads: http://apl.aip.org/features/most_downloaded

Information for Authors: http://apl.aip.org/authors

\section{ADVERTISEMENT}
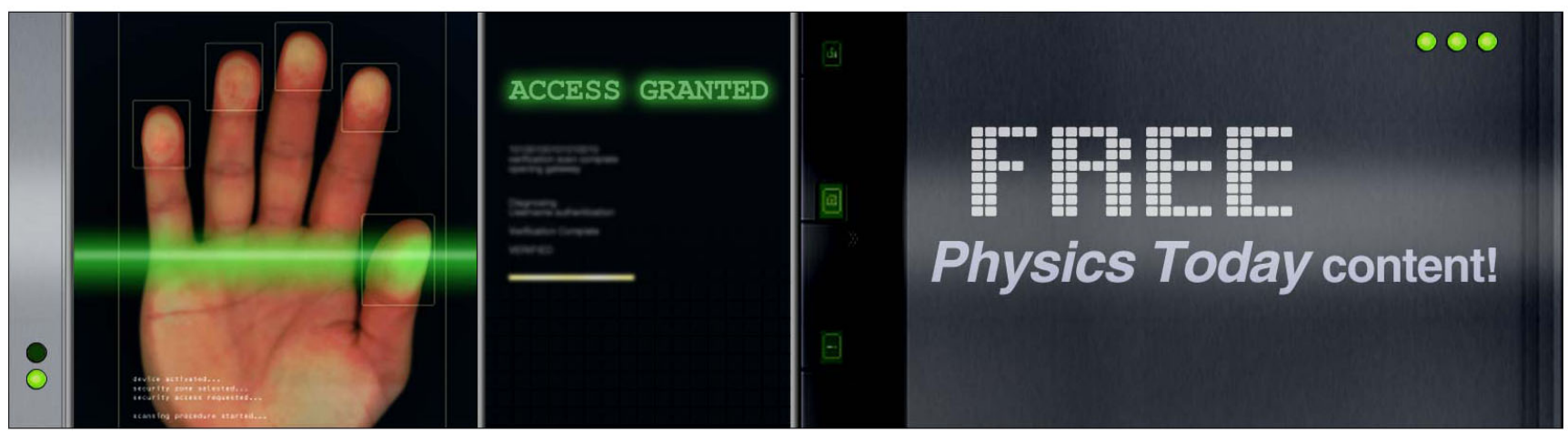


\title{
Spin-flip phenomena at the Co|graphene|Co interfaces
}

\author{
Baihai Li, ${ }^{1,2}$ Liang Chen, ${ }^{1, a)}$ and Xiaoqing Pan $^{2}$ \\ ${ }^{1}$ Institute of Materials Technology and Engineering, Chinese Academy of Sciences, Ningbo, \\ Zhejiang 315201, People's Republic of China \\ ${ }^{2}$ Department of Materials Science and Engineering, University of Michigan, Ann Arbor, \\ Michigan 48109, USA
}

(Received 4 January 2011; accepted 9 March 2011; published online 30 March 2011)

\begin{abstract}
In the present letter, we report phenomena at the Co|Graphene|Co interfaces based on first principles calculations. The nature of $\mathrm{Co}-\mathrm{C}-\mathrm{Co}$ and $\mathrm{Co}-$ graphene-Co atomic bonding at the interfaces were investigated by means of density of states, electron and spin density analysis. We show that the spins are antiferromagnetic coupled across the interface via the $p d-\pi$ interactions between the Gr and Co layers, as exemplified by the superexchange mechanism. As a consequence, the spin injection efficiency at the $\mathrm{Co} \mid$ Graphene|Co interfaces and junctions will be reduced. (C) 2011 American Institute of Physics. [doi:10.1063/1.3571553]
\end{abstract}

Successful preparation of graphene (Gr) (Refs. 1 and 2) have attracted tremendous interest in realizing the potential application of $\mathrm{Gr}$ in spintronic devices. ${ }^{3,4}$ The well-fitting in-plane geometric structures and Fermi surfaces of Gr with transition metals $(\mathrm{TMs}=\mathrm{Co}, \mathrm{Ni}, \mathrm{Cu})$ surfaces are assumed to be helpful to yield remarkable magnetoresistance (MR) effect. ${ }^{5,6}$ Indeed, spin injection from ferromagnetic metal into Gr has been realized, ${ }^{3,7,8}$ however, the efficiency is rather limited $(\sim 10 \%){ }^{8,9}$ That is mainly attributed to the conductivity mismatch between $\mathrm{Gr}$ and ferromagnetic metals. ${ }^{10}$ Nevertheless, the coupling interactions between two electrodes could remarkably influence the spin transmission process throughout the spin filter junction. However, pertinent study is sparse. In this letter, we chose Co as the electrode to unravel the nature of interactions in the system. Interestingly, our calculations show some distinctive features of electronic structures at the $\mathrm{Co}|\mathrm{Gr}| \mathrm{Co}$ interfaces, namely, the interfacial metal atoms prefer antiferromagnetic coupling via the intercalated Gr layer.

Spin polarized density functional theory (DFT) calculations were performed using the generalized gradient approximation (GGA) Perdew-Wang functional (PW91) in this study. ${ }^{11}$ The electron-core interactions were treated using the projector-augmented-wave method. ${ }^{12}$ Similar methods have been widely employed to study the graphene/metal interfaces. ${ }^{13-15}$ A $14 \AA$ vacuum region perpendicular to the surface were used. All atoms were fully relaxed with the forces converged to less than $0.02 \mathrm{eV} / \AA$.

In agreement with previous studies, our calculations indicate that the most stable configuration for Gr layer on Co slab is $\mathrm{Gr} \mid \mathrm{Co}_{\mathrm{AC}}$, in which half of $\mathrm{C}$ atoms of the Gr layer reside above the outmost $\mathrm{Co}$ atoms (site $\mathrm{A}$ ) and the rest of $\mathrm{C}$ atoms reside above the third layer Co atoms (site C). ${ }^{16,17}$ The calculated binding energy (21.5 meV/atom) of the $\mathrm{Gr} \mid \mathrm{Co}_{\mathrm{AC}}$ structure is comparable with the value (30 meV/atom), where the van de Waals contributions were considered, as shown in Ref. 18.

Deposition of a single Co atom on the Co slab supported Gr layer is the precursor to fabricate the $\mathrm{Co}|\mathrm{Gr}| \mathrm{Co}$ spin filtering junction. Three deposited positions on top of $\mathrm{Gr} \mid \mathrm{Co}_{\mathrm{AC}}$

${ }^{a)}$ Electronic mail: chenliang@nimte.ac.cn. were considered, as shown in Fig. 1(a). The single Co atom deposited on the sixfold $\mathrm{B}^{*}$ site $\left(\mathrm{Co}_{\mathrm{B}^{*}}^{\mathrm{S}} / \mathrm{Gr} \mid \mathrm{Co}_{\mathrm{AC}}\right)$ yields the highest binding energy of $1.63 \mathrm{eV}$. The one-fold $\mathrm{C}^{*}$ site is less favorable with a binding energy of $1.23 \mathrm{eV}$. The $\mathrm{A}^{*}$ site is an unstable position, where the initially deposited Co atom will migrate to the $\mathrm{B}^{*}$ site after optimization. Bader charge analysis $^{19}$ indicates that 0.48 and 0.24 electrons were donated by the deposited $\mathrm{Co}$ atoms to their coordinated $\mathrm{C}$ atoms for the $\mathrm{Co}_{\mathrm{B}^{*}}^{\mathrm{S}} / \mathrm{Gr} \mid \mathrm{Co}_{\mathrm{AC}}$ and $\mathrm{Co}_{\mathrm{C}^{*}}^{\mathrm{S}} / \mathrm{Gr} \mid \mathrm{Co}_{\mathrm{AC}}$ structures, respectively. Interestingly, it is unexpected that the spin orientation of the deposited Co atom is antiparallel to the supporting Co slab. Energetically, the antiferromagnetic coupling configurations are 0.30 and $0.06 \mathrm{eV}$ lower than the ferromagnetic alignments in total energies for $\mathrm{Co}_{\mathrm{B}^{*}}^{\mathrm{S}} / \mathrm{Gr} \mid \mathrm{Co}_{\mathrm{AC}}$ and $\mathrm{Co}_{\mathrm{C}^{*}}^{\mathrm{S}} / \mathrm{Gr} \mid \mathrm{Co}_{\mathrm{AC}}$, respectively. Correspondingly, the magnetic moments of the deposited Co atoms are calculated to be $-1.62 \mu_{\mathrm{B}}$ and $-2.21 \mu_{\mathrm{B}}$ for the $\mathrm{Co}_{\mathrm{B}^{*}}^{\mathrm{S}} / \mathrm{Gr} \mid \mathrm{Co}_{\mathrm{AC}}$ and $\mathrm{Co}_{\mathrm{C}^{*}}^{\mathrm{S}} / \mathrm{Gr} \mid \mathrm{Co}_{\mathrm{AC}}$, respectively. The magnetic moments of the supporting layer are calculated to be 1.56-1.67 $\mu_{\mathrm{B}}$.

The spin-flip phenomenon also occurs when Co monolayer is deposited on $\mathrm{Gr} \mid \mathrm{Co}_{\mathrm{AC}}$. The stability of the interfaces

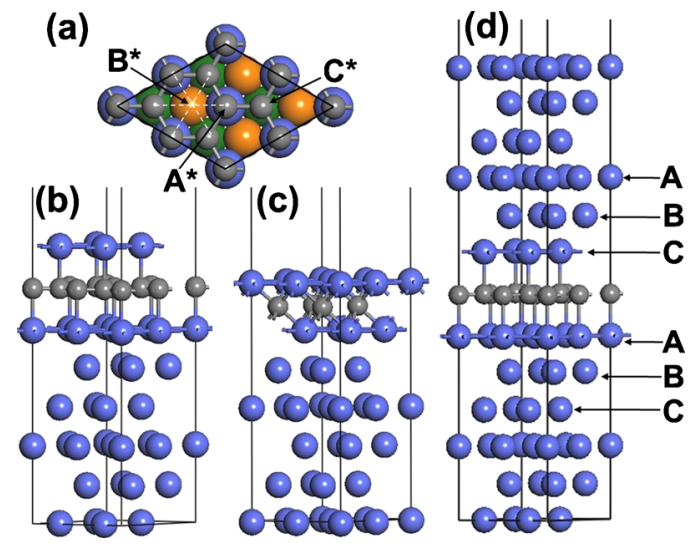

FIG. 1. (Color online) (a) Top view of the deposited positions for a single cobalt atom on the supported Gr layer: $\mathrm{A}^{*}$ and $\mathrm{C}^{*}$ sites are above the carbon atoms that are on top of the outmost Co layer (blue) and the third Co layer (green), $\mathrm{B}^{*}$ is above the center of the $\mathrm{C}$-ring and on top of the second layer (orange) of the supporting Co slab; (b) side view of the $\mathrm{Co}_{\mathrm{C} *}^{\mathrm{L}} / \mathrm{Gr} \mid \mathrm{Co}_{\mathrm{AC}}$ interface; (c) side view of the $\mathrm{Co}^{\mathrm{C} \text {-layer }}$ structure; (d) side view of the $\mathrm{Co}_{\mathrm{CA}}|\mathrm{Gr}| \mathrm{Co}_{\mathrm{AC}}$ interfaces. 


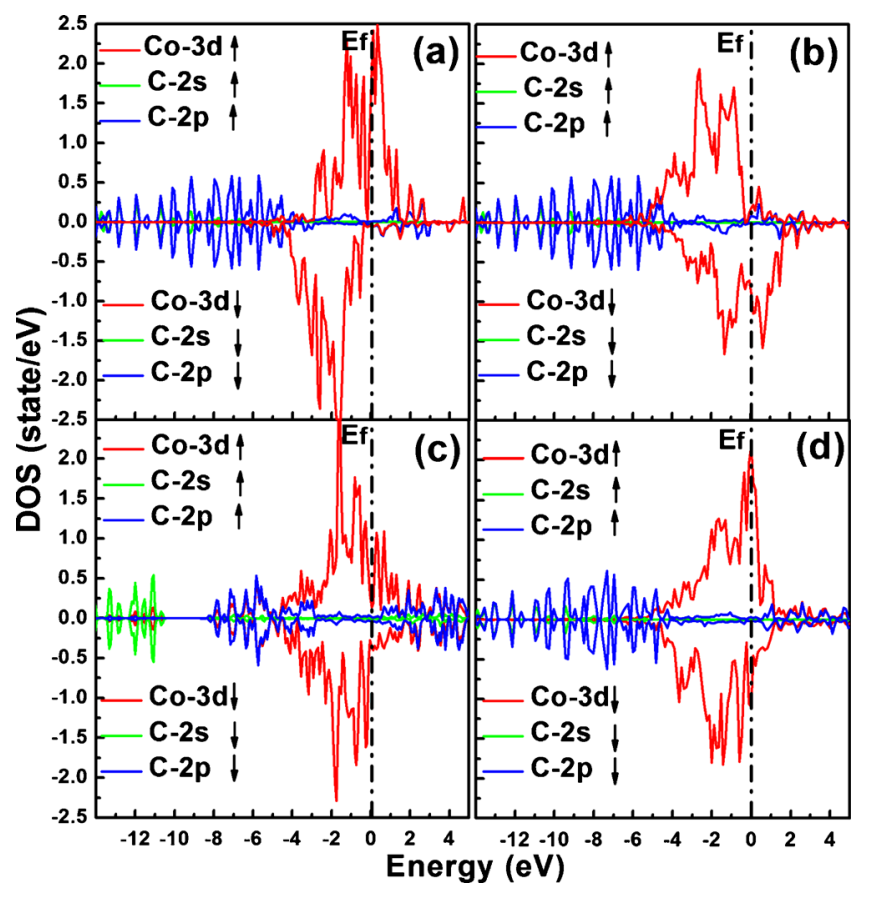

FIG. 2. (Color online) (a) The $3 d$ states of a Co atom of the deposited Co monolayer and the $2 s, 2 p$ states of a $\mathrm{C}$ atom in the $\mathrm{Gr}$ layer of $\mathrm{Co}_{\mathrm{C} *}^{\mathrm{L}} / \mathrm{Gr} \mid \mathrm{Co}_{\mathrm{AC}}$; (b) The $3 d$ states of a Co atom underneath the Gr layer and the $2 s, 2 p$ states of a $\mathrm{C}$ atom in the Gr layer of $\mathrm{Co}_{\mathrm{C} *}^{\mathrm{L}} / \mathrm{Gr} \mid \mathrm{Co}_{\mathrm{AC}}$; (c) The $3 d$ states of a Co atom of the outmost layer and the $2 s, 2 p$ states of the scattered $\mathrm{C}$ atom of $\mathrm{Co}^{\mathrm{C}-\text { layer; }}$; (d) The $3 d$ states of a Co atom of the upper electrode bonding to the intercalated $\mathrm{Gr}$ layer and the $2 s, 2 p$ states of a $\mathrm{C}$ atom in the Gr layer of $\mathrm{Co}_{\mathrm{CA}}|\mathrm{Gr}| \mathrm{Co}_{\mathrm{AC}}$. The Fermi levels are shifted to zero eV.

is justified by the interfacial work of separation $\left(\mathrm{W}_{\text {sep }}\right)$,

$$
\mathrm{W}_{\text {sep }}=\left(\mathrm{E}_{\mathrm{Co}-\text { layer }}+\mathrm{E}_{\mathrm{Co} \text {-slab }}+\mathrm{E}_{\mathrm{Gr}}-\mathrm{E}_{\mathrm{Co}} \mathrm{L}_{/ \mathrm{Gr}} \mathrm{Co}_{\mathrm{AC}}\right) / 2 \mathrm{~A} \text {, }
$$

where $\mathrm{E}_{\mathrm{Co}^{\mathrm{L}} / \mathrm{GrlCo}_{A C}}$ is the total energy of one Co monolayer on $\mathrm{Gr} \mid \mathrm{Co}_{\mathrm{AC}}, \mathrm{E}_{\mathrm{Co} \text {-slab }}, \mathrm{E}_{\mathrm{Co} \text {-layer, }}$, and $\mathrm{E}_{\mathrm{Gr}}$ are the total energies of the isolated Co slab, Co monolayer, and $\mathrm{Gr}$ contained in the same cell. A is the surface area of the interface. The $\mathrm{C}^{*}$ site is the preferential site for the individual $\mathrm{Co}$ atom within the monolayer with $\mathrm{W}_{\text {sep }}$ of $1.22 \mathrm{~J} / \mathrm{m}^{2}$, denoted as $\mathrm{Co}_{\mathrm{C} *}^{\mathrm{L}} / \mathrm{Gr} \mid \mathrm{Co}_{\mathrm{AC}}$ and shown in Fig. 1(b). The $\mathrm{A}^{*}$ and $\mathrm{B}^{*}$ sites are unfavorable positions to anchor the Co monolayers, which are pushed away with $\mathrm{C}-\mathrm{Co}$ distances longer than 3.2 $\AA$. The strong cohesive interactions $(5.53 \mathrm{eV} / \mathrm{atom})$ within the deposited Co monolayer significantly weaken the interactions with the supporting Gr layer (the binding energy is calculated to be $0.06 \mathrm{eV} /$ atom). Nevertheless, the deposited Co monolayer still prefers antiferromagnetic coupling with the supporting Co slab via the sandwiched Gr layer, with the magnetic moment of $-1.71 \mu_{\mathrm{B}}$. The total energy of antiferromagnetic coupling configuration is $0.42 \mathrm{eV}$ lower than that of ferromagnetic coupling alignment.

The density of states (DOS) of a Co atom in the deposited monolayer and its coordinated $\mathrm{C}$ atom are shown in Fig. 2(a). The Co-C $p d-\pi$ bonding interactions between Co $3 d$ and $\mathrm{C} 2 s,-2 p$ orbitals $[(-14)-(-4) \mathrm{eV}]$ are rather weak. While the Co $3 d$ spin-up states are significantly pushed up to the higher energy level, and the spin-down states are shift down, which result in the spin-down magnetism of this Co atom. For comparison, the DOS of a Co atom underneath the Gr layer and its coordinated C atom are shown in Fig. 2(b). The area of the spin-up states below the Fermi level is considerably larger than that of the spin-down states, thus yield-

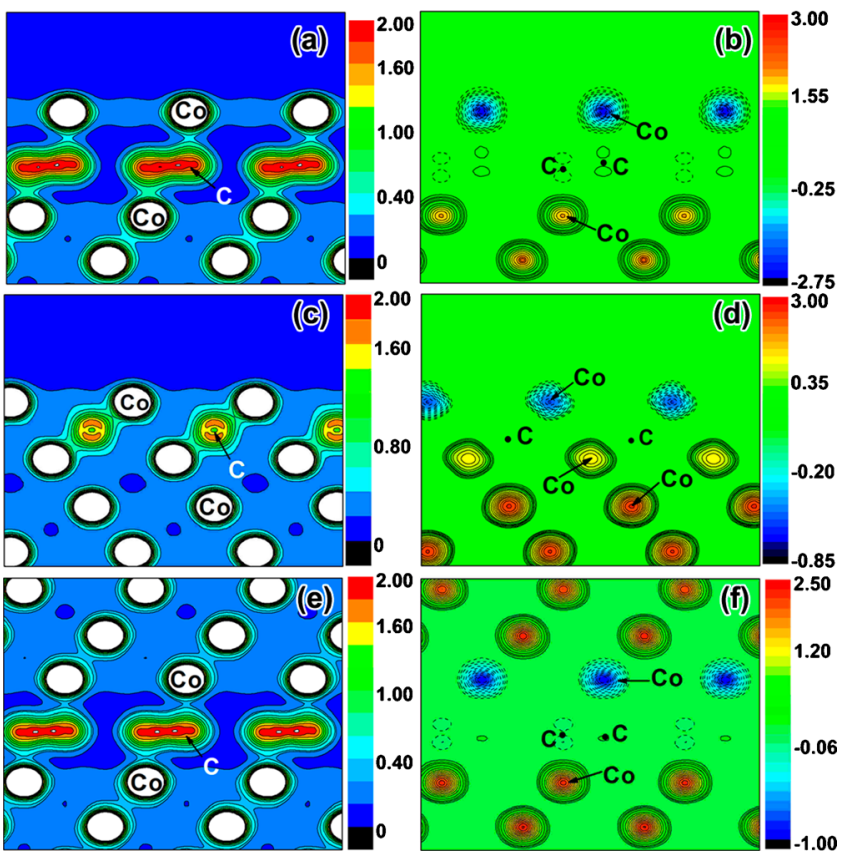

FIG. 3. (Color online) (a), (c), (e) are the charge density and (b), (d), (f) are the spin density of the (110) cut of $\mathrm{Co}_{\mathrm{C} *}^{\mathrm{L}} / \mathrm{Gr} \mid \mathrm{Co}_{\mathrm{AC}}, \mathrm{Co}^{\mathrm{C} \text {-layer }}$ and $\mathrm{Co}_{\mathrm{CA}}|\mathrm{Gr}| \mathrm{Co}_{\mathrm{AC}}$ structures, respectively. The solid and dash lines in the spin density maps represent spin-up and spin-down, respectively.

ing the spin-up magnetism. That is, the deposited Co monolayer prefers antiferromagnetic coupling with the supporting Co layer via the intercalated Gr layer.

The electron and spin density images are useful to visualize the nature of the bonding and magnetic coupling interactions. In Fig. 3(a), the strong $s p^{2}-\sigma$ bonding within the supported Gr layer shows much higher electron density than that of $p d-\pi$ bonding between $\mathrm{C} 2 p_{z}$ and Co $3 d$ orbitals. Note that the contour with density higher than $2.0 \mathrm{e} / \AA^{3}$ is not shown. Via the $p d-\pi$ bonding, electrons are transferred from Co atoms to the Gr layer and then occupy the $s p^{2}-\sigma^{*}$ antibonding state. The spin orientations of the transferred electrons are not changed in this process. As a result, the unpaired electrons lead to slight polarization of the $\mathrm{C}$ atoms in the Gr layer $\left(\sim \pm 0.05 \mu_{\mathrm{B}}\right)$ with the spin lobes antiparallel to each other [see Fig. 3(b)].

According to the superexchange mechanism proposed by Goodenough and Slater, ${ }^{20,21}$ the spin orientations of $\mathrm{C}$ and Co atoms should be antiparallel to each other, otherwise, the electron hopping can't occur. Indeed, as shown in Fig. 3(b), the supporting Co slab is aligned in ferromagnetic ordering with the same spin orientation (solid line) while the mediated Gr layer leads the deposited Co monolayer to be antiparallel (dash line) with respect to the supporting Co slab. On the other hand, the itinerant electrons within the intercalated $\mathrm{Gr}$ layer mediated Ruderman-Kittel-Kasuya-Yosida (RKKY) interactions are assumed to be an alternative exchange mechanism. However, the effect of the superexchange mechanism on the antiferromagnetic coupling should predominate over the RKKY mechanism in this structure, as demonstrated in the below.

We next placed $\mathrm{C}$ atoms on all the octahedral sites of the first sublayer of the Co(111) slab to form a "discrete" layer (with half the number of atoms of the Gr layer), denoted as $\mathrm{Co}^{\mathrm{C}-\text { layer }}$ and shown in Fig. 1(c). The interactions of the $\mathrm{C}$ atoms with Co slab are calculated to be $4.50 \mathrm{eV} /$ atom. The 
$\mathrm{C}-\mathrm{C}$ interactions are significantly weakened $(2.18 \mathrm{eV} /$ atom $)$ and the delocalized conduction electrons are almost eliminated in the intercalated C layer. The DOS shown in Fig. 2(c) indicates that the $3 d$ states of an outmost Co atom are strongly hybridized with the $2 s, 2 p$ states of its coordinated $\mathrm{C}$ atom in the ranges of $(-14)-(-10) \mathrm{eV}$ and $(-8)-(-3) \mathrm{eV}$, respectively. Hence, the spin-up states are pushed up to higher energy region while the spin-down states are shifted down, resulting in the area of the spin-up states under the Fermi level is turned to be smaller than that of the spin-down states. As a result, the spin orientation of the outmost Co layer is flipped, yielding a magnetic moment of $-0.48 \mu_{\mathrm{B}}$. The magnetic moment of the lower Co layers is calculated to be $0.45 \mu_{\mathrm{B}}$. Figure 3(c) clearly displays the $s p^{3}-d^{2}$ bonding interactions of the inserted $\mathrm{C}$ atoms with Co layers, which are considerably stronger than the $p d-\pi$ bonding interactions between Co layers and the intercalated Gr layer. Furthermore, as shown in Fig. 3(d), the spin orientation of the outmost Co layer is antiparallel to the lower Co layers. The spin lobes of $\mathrm{C}$ atoms do not appear due to the negligible magnetic moments $\left(\sim 0.02 \mu_{\mathrm{B}}\right)$. Apparently, the antiferromagnetic coupling of the outmost and lower Co layers is a typical pattern of the superexchange interactions. Therefore, RKKY coupling plays a trivial role in the single Gr layer intercalated structures, compared to the superexchange mechanism.

Finally, we investigate the electronic structures of the sandwiched $\mathrm{Co}|\mathrm{Gr}| \mathrm{Co}$ spin filtering junction, where a $\mathrm{Gr}$ layer is intercalated between two six-layer $\mathrm{Co}(111)$ slabs. Half of $\mathrm{C}$ atoms of the Gr layer are located on the A sites bonding to the outmost $\mathrm{Co}$ atoms of the lower electrode and the rest of $\mathrm{C}$ atoms form bonding with the lowest Co atoms (site $\mathrm{C}$ ) of the upper electrode to lower the total energy, which is denoted as $\mathrm{Co}_{\mathrm{CA}}|\mathrm{Gr}| \mathrm{Co}_{\mathrm{AC}}$ and shown in Fig. 1(d). $\mathrm{W}_{\text {sep }}$ is calculated to be $1.13 \mathrm{~J} / \mathrm{m}^{2}$ according to Eq. (1) in which $\mathrm{E}_{\text {Co-layer }}$ is replaced by $\mathrm{E}_{\mathrm{Co-slab}}$. The magnetic moments are calculated to be -0.65 and $1.45 \mu_{\mathrm{B}}$ for the upper and lower interfacial Co layers, respectively. In contrast, the magnetic moments of other Co atoms are still 1.31-1.68 $\mu_{\mathrm{B}}$. The Gr layer has rather limited magnetic moment of $\pm 0.03 \mu_{\mathrm{B}}$. The DOS of an upper interfacial Co atom and its bonding $\mathrm{C}$ atom is shown in Fig. 2(d), where the area of spin-down states under the Fermi level is larger than that of spin-up states. The electron density shown in Fig. 3(e) also indicates that the $p d-\pi$ interactions of the interfacial Co atoms with the intercalated Gr layer are rather weak. Likewise, the sandwiched structure yields antiferromagnetic coupling of the interfacial Co atoms via the mediated Gr layer, as shown in Fig. 3(f).

The antiferromagnetic coupling at the interfaces of the junction might have important influence on the spindependent transmission through this junction. The transmission probability of an incoming electron current is dependent on the available DOS with the same spin orientation in both electrodes. ${ }^{22}$ The antiparallel coupling at the $\mathrm{Co}|\mathrm{Gr}| \mathrm{Co}$ interfaces may change the spin polarization of the conductance when an external magnetic field is applied and leads to low efficient transmission MR, according to Julliere's formula ${ }^{23}$

$$
\mathrm{MR}=\frac{2 P_{1} P_{2}}{1-P_{1} P_{2}}, \quad P_{i}=\frac{\rho_{i}^{\uparrow}-\rho_{i}^{\downarrow}}{\rho_{i}^{\uparrow}+\rho_{i}^{\downarrow}} \quad(i=1,2),
$$

where the effective spin polarization $\left(P_{i}\right)$ is dependent on the density of the majority $\left(\rho_{i}^{\uparrow}\right)$ and minority $\left(\rho_{i}^{\downarrow}\right)$ states at the
Fermi level, respectively. Taking thick graphite film to decouple the short-range antiferromagnetic interactions at the interfaces, might be a feasible solution to enhance the MR effect, as demonstrated in Refs. 5 and 6. Our calculations indicated the parallel coupling is preferential at the interfaces with $0.14 \mathrm{eV}$ lower in total energy than antiparallel configuration when two layers of $\mathrm{Gr}$ intercalated between two electrodes. The magnetic moments are calculated to be $1.56 \mu_{\mathrm{B}}$ for both the upper and lower interfacial Co layers.

In conclusion, we found an interesting spin-flip phenomenon at the $\mathrm{Co}|\mathrm{Gr}|$ Co interfaces from first principles. The detailed analysis of the DOS and the spin density maps at the interfaces indicates that the superexchange mechanism predominates over the ferromagnetic RKKY coupling. As a consequence, the antiferromagnetic coupling takes place at the Co-Gr interfaces via the $p d-\pi$ interactions between the $\mathrm{Gr}$ and Co layers.

The work is financially supported by the technology innovative research program of Ningbo Municipality and Ningbo Science Foundation (2009A610124).

${ }^{1}$ K. S. Novoselov, A. K. Geim, S. V. Morozov, D. Jiang, Y. Zhang, S. V. Dubonos, I. V. Grigorieva, and A. A. Firsov, Science 306, 666 (2004).

${ }^{2}$ C. Berger, Z. Song, X. Li, X. Wu, N. Brown, C. Naud, D. Mayou, T. Li, J. Hass, A. N. Marchenkov, E. H. Conrad, P. N. First, and W. A. de Heer, Science 312, 1191 (2006).

${ }^{3}$ N. Tombros, C. Jozsa, M. Popinciuc, H. T. Jonkman, and B. J. van Wees, Nature (London) 448, 571 (2007).

${ }^{4}$ Y. B. Zhang, Y. W. Tan, H. L. Stormer, and P. Kim, Nature (London) 438, 201 (2005).

${ }^{5}$ V. M. Karpan, G. Giovannetti, P. A. Khomyakov, M. Talanana, A. A. Starikov, M. Zwierzycki, J. van den Brink, G. Brocks, and P. J. Kelly, Phys. Rev. Lett. 99, 176602 (2007).

${ }^{6}$ V. M. Karpan, P. A. Khomyakov, A. A. Starikov, G. Giovannetti, M. Zwierzycki, M. Talanana, G. Brocks, J. van den Brink, and P. J. Kelly, Phys. Rev. B 78, 195419 (2008).

${ }^{7}$ M. Ohishi, M. Shiraishi, R. Nouchi, T. Nozaki, T. Shinjo, and Y. Suzuki, Jpn. J. Appl. Phys., Part 2 46, L605 (2007).

${ }^{8}$ E. W. Hill, A. K. Geim, K. Novoselov, F. Schedin, and P. Blake, IEEE Trans. Magn. 42, 2694 (2006).

${ }^{9}$ L. Brey and H. A. Fertig, Phys. Rev. B 76, 205435 (2007)

${ }^{10}$ G. Schmidt, D. Ferrand, L. W. Molenkamp, A. T. Filip, and B. J. van Wees, Phys. Rev. B 62, R4790 (2000).

${ }^{11}$ J. P. Perdew, K. Burke, and M. Ernzerhof, Phys. Rev. Lett. 77, 3865 (1996).

${ }^{12}$ P. E. Blöchl, Phys. Rev. B 50, 17953 (1994).

${ }^{13}$ J. C. W. Swart, E. van Steen, I. M. Ciobica, and R. A. van Santen, Phys. Chem. Chem. Phys. 11, 803 (2009).

${ }^{14}$ Q. S. Ran, M. Z. Gao, X. M. Guan, Y. Wang, and Z. P. Yu, Appl. Phys. Lett. 94, 103511 (2009).

${ }^{15}$ D. E. Jiang, M. H. Du, and S. Dai, J. Chem. Phys. 130, 074705 (2009).

${ }^{16}$ G. Giovannetti, P. A. Khomyakov, G. Brocks, V. M. Karpan, J. van den Brink, and P. J. Kelly, Phys. Rev. Lett. 101, 026803 (2008).

${ }^{17}$ D. Eom, D. Prezzi, K. T. Rim, H. Zhou, M. Lefenfeld, S. Xiao, C. Nuckolls, M. S. Hybertsen, T. F. Heinz, and G. W. Flynn, Nano Lett. 9, 2844 (2009).

${ }^{18}$ M. Vanin, J. J. Mortensen, A. K. Kelkkanen, J. M. Garcia-Lastra, K. S. Thygesen, and K. W. Jacobsen, Phys. Rev. B 81, 081408(R) (2010).

${ }^{19}$ R. F. W. Bader, Atoms in Molecules: A Quantum Theory (Oxford University Press, New York, 1990).

${ }^{20}$ J. B. Goodenough, Phys. Rev. 100, 564 (1955).

${ }^{21}$ J. C. Slater, Quart. Progr. Rep. MIT 1, July 15; October 15, 1953.

${ }^{22}$ J. M. De Teresa, Physics 2, 13 (2009).

${ }^{23}$ M. Julliere, Phys. Lett. A 54, 225 (1975). 